\title{
Adaptive Dynamic Surface Control: Stability Analysis Based on LMI
}

\author{
Bongsob Song
}

Department of Mechanical Engineering, Ajou University, Suwon, 443-749, Korea (ROK)

\begin{abstract}
In this paper, quadratic stability of adaptive dynamic surface control for a class of nonlinear systems in strict-feedback form is analyzed in the framework of linear matrix inequality. While the existence of controller gains and filter time constants for semi-global stability was theoretically proved in the literature, it is not sufficient to describe how a set-point value and parameter update laws affect stability and parameter convergence. Thus, it is necessary to provide a systematic analysis method to guarantee both stability and parameter convergence. By deriving the augmented closed-loop error dynamics in linear differential inclusion form, a sufficient condition of the controller gains for stability and parameter convergence is derived in the form of linear matrix inequality. Finally, the quadratic Lyapunov function for its quadratic stability is computed numerically via convex optimization.
\end{abstract}

Keywords Adaptive Dynamic Surface Control, Quadratic Stability, Parameter Convergence, Linear Matrix Inequality

\section{Introduction}

The dynamic surface control (DSC) is a dynamic extension of multiple sliding surface (MSS) control to overcome the drawback of "explosion of complexity" in backstepping as well as MSS control[1]. The use of a series of dynamic filters enables the controller to be designed sequentially and simple. Furthermore, the existence of controller gains for semi-global stability was theoretically proved in[1]. Recently, an analysis and design method in the framework of convex optimization has been introduced to allow us to find a quadratic Lyapunov function numerically for a class of nonlinear systems called strict-feedback form[2].

This control approach was extended to a class of nonlinear systems where the uncertainty is linearly parameterized, e.g., $a f_{1}$ in (1) where $a$ is an unknown constant and $f_{1}$ is a known nonlinear function. The adaptive backstepping method has been developed[3] and extended to a class of time-delay nonlinear systems[4, 5]. As introduced above, the adaptive DSC to solve the problem of "explosion of complexity" has been developed for a class of nonlinear systems and time-delay systems[6, 7]. Furthermore, DSC has been combined with adaptive neural network control scheme in the literature [8,9]. However, the useful tools such as linear matrix inequalities (LMIs) are hard to apply to nonlinear system with linearly parameterized uncertainties. There is little work in the literature for LMIs to be used for stability analysis of adaptive nonlinear control problems.

* Corresponding author:

bsong@ajou.ac.kr (Bongsob Song)

Published online at http://journal.sapub.org/control

Copyright (C) 2012 Scientific \& Academic Publishing. All Rights Reserved
The following example illustrates the design procedure of adaptive dynamic surface control in[6]:

$$
\begin{aligned}
& \dot{x}_{1}=x_{2}-a x_{1}^{2}:=x_{2}+a f_{1}\left(x_{1}\right) \\
& \dot{x}_{2}=u-x_{2}
\end{aligned}
$$

where $a$ is unknown but bounded by a known positive constant $c$ such that $a \in[0, c]$ and $f_{1}=-x_{1}^{2}$ is a known nonlinear function and locally Lipschitz on $D=\left\{x \in \mathfrak{R}^{2} \mid\|x\|<r, r>0\right\}$, i.e.,

$$
\left|\frac{\partial f_{1}}{\partial x_{1}}\right|=\left|-2 x_{1}\right| \leq 2 r:=\gamma
$$

where $\gamma$ is a Lipschitz constant. The control objective is $x_{1}(t) \rightarrow x_{1 d}$ where $x_{1 d}$ is a constant with $\dot{x}_{1 d}=\ddot{x}_{1 d}=0$, which is called a set-point control problem.

First, define the first error surface as $S_{1}=x_{1}-x_{1 d}$. After taking its derivative along the trajectories of (1)

$$
\dot{S}_{1}=x_{2}+a f_{1},
$$

the synthetic input, which is to drive $S_{1} \rightarrow 0$, is

$$
\bar{x}_{2}=-\hat{a} f_{1}-K S_{1}
$$

where $K$ is a controller gain and $\hat{a}$ is the estimate of the unknown parameter $a$ following the update law as propose in[6]:

$$
\dot{\hat{a}}=\rho S_{1} f_{1}
$$

where $\rho$ is a positive constant.

Then, define the second sliding surface as $S_{2}=x_{2}-x_{2 d}$ where $x_{2 d}$ equals $\bar{x}_{2 d}$ passed through a first-order filter, i.e.,

$$
\tau \dot{x}_{2 d}+x_{2 d}=\bar{x}_{2}, x_{2 d}(0)=\bar{x}_{2}(0)
$$

where $\tau$ is a filter time constant. Similarly, the derivative of $S_{2}$ along the trajectories of (1) is 


$$
\dot{S}_{2}=\dot{x}_{2}-\dot{x}_{2 d}=u-x_{2}-\dot{x}_{2 d},
$$

and the control input is derive as

$$
u=\dot{x}_{2 d}+x_{2}-K S_{2}
$$

where $\dot{x}_{2 d}$ is calculated from (4) such that

$$
\dot{x}_{2 d}=\frac{\bar{x}_{2}-x_{2 d}}{\tau} \text {. }
$$

It is interesting to remark that the calculation of $\dot{x}_{2 d}$ becomes simpler due to the inclusion of the first-order filter while it results in "explosion of complexity" in backstepping.

A next question is how to design a set of controller gains to guarantee stability, e.g., $K, \tau$ and $\rho$ in the example. It was proven in[6] that there exist a set of controller gains ( $K$ and $\tau$ ) to guarantee the stability for stabilization and set-point control problems. However, the performance of the adaptive DSC depends on $\rho$ critically[10]. If a small magnitude of $\rho$ is chosen, the adaption of $a$ in (3) will be slow and the transient error will be large. On the other hand, too large magnitude of $\rho$ will lead to oscillatory estimation of the parameter, thus resulting in the oscillatory error.

Suppose $a=1$ in (1), $K=2.5$ in (2) and (5), and $\tau=0.05$ in (4). When $\rho$ is assigned as 1 and 10 respectively, the time responses of $x_{1}$ and $\hat{a}$ are shown is Fig. 1. As explained above, the larger magnitude of $\rho$ results in faster convergence of estimation error of $\hat{a}$ and tracking error. However, when $\rho=70$, the oscillatory estimation of the parameter is shown in Fig. 1. Thus, the tracking error does not converge to zero. Furthermore, if $x_{1 d}$ is changed to a different constant, although it will be discussed later in Section 4, the different time response (e.g., oscillatory estimation) of $\hat{a}$ may be shown for the same set of $K$, $\tau$, and $\rho$.
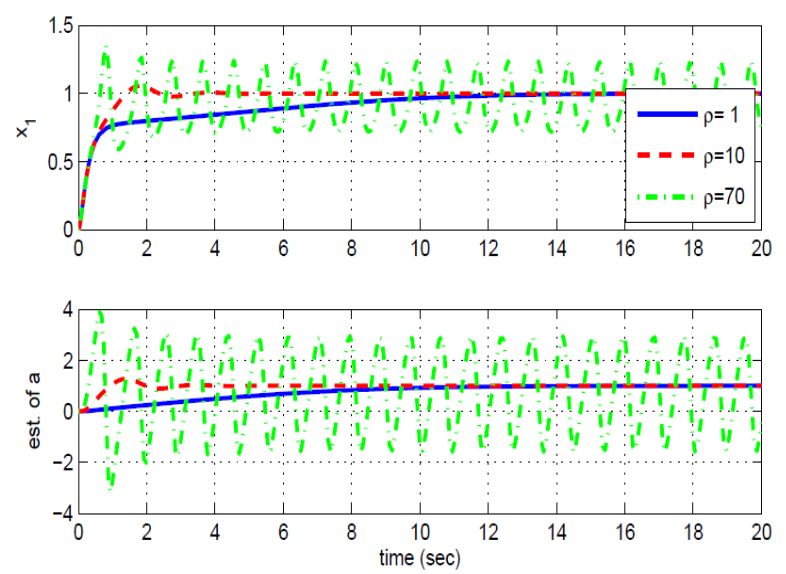

Figure 1. Time response of $x_{1}$ and estimate of $a$ with respect to $\rho$

Motivated by this example, it is unclear what values of $\rho$ and $x_{1 d}$ guarantee stability and convergence of the parameter estimation error for the given set of a controller gain $(K)$ and a time constant $(\tau)$. The main contribution of this paper is to derive the augmented closed-loop error dynamics including parameter estimation errors and filter errors in linear differential inclusion form, and to derive the sufficient condition for stability and parameter convergence. Fur- thermore, the sufficient condition allows us to check stability of the closed-loop system and convergence of estimated parameters by solving the LMI numerically.

Through this paper, we will use the following notation: $0_{n} \in \mathfrak{R}^{n}$ is a zero vector and $0 \in \mathfrak{R}^{m \times n}$ is a zero matrix with appropriate dimensions. $I_{n} \in \mathfrak{R}^{n \times n}$ is a square identity matrix and $I_{m \times n} \in \mathfrak{R}^{m \times n}$ is an identity matrix in the sense that all diagonal elements are one whatever the dimension of the matrix is. If $x_{n} \in \mathfrak{R}^{n}$ is a vector, $\operatorname{diag}(x)$ is a diagonal matrix with the vector $x$ forming the diagonal and $\operatorname{diag}(x, i)$ (or $\operatorname{diag}(x,-i))$ is a square matrix of size $(n+i)$ with the vector $x$ forming the $i$ th super-diagonal (or sub-diagonal) stands for a positive definite (or semidefinite) matrix, $\operatorname{Tr}(X)$ is the sum of all diagonal entries in $X$.

\section{Problem Statement}

Consider the class of nonlinear system as follows:

$$
\left\{\begin{array}{l}
\dot{x}_{i}=\dot{x}_{i+1}+a_{i} f_{i}\left(x_{1}, \ldots, x_{i}\right), \quad i=1, \ldots, n-1 \\
\dot{x}_{n}=u+a_{n} f_{n}\left(x_{1}, \ldots, x_{n}\right)
\end{array}\right.
$$

where $a_{i}$ is an unknown parameter but bounded by a known positive constant $c_{i}$ such that $a_{i} \in\left[\begin{array}{ll}0 & c_{i}\end{array}\right], f_{i}$ and $\left[\partial f_{i} / \partial x\right]$ are continuous on $D \in \Re^{n}$ and $f_{i}: D_{i} \rightarrow \mathfrak{R}$ on $D_{i} \subset D$ is a known nonlinear function in strict-feedback form in the sense that the $f_{i}$ depend only on $x_{1}, \ldots, x_{i}$. It is implied that $f_{i}$ is locally Lipschitz and $\left[\partial f_{i}(x) / \partial x\right]$ is bounded on $D_{i}[11]$. Therefore, there exists a constant $\gamma_{i}>0$ such that

$$
\left\|\frac{\partial f_{i}}{\partial x}\right\|=\left\|\left[\frac{\partial f_{i}}{\partial x_{1}} \cdots \frac{\partial f_{i}}{\partial x_{i}}\right]\right\| \leq \gamma_{i}
$$

for all $x$ on $D_{i}$.

\section{Adaptive Dynamic Surface Control}

\subsection{Design Procedure}

An outline of the standard design procedure for the adaptive DSC described in $[6,12]$ is as follows: Define the $i$ th error surface as $S_{i}:=x_{i}-x_{i d}$ for $i=1, \ldots, n$ where $x_{1 d}$ is the constant value. After taking the time derivative of $S_{i}$ along the trajectories of (6),

$$
\dot{S}_{i}:=a_{i} f_{i}+x_{i+1}
$$

The surface error $S_{i}$ will converge to zero if $S_{i} \dot{S}_{i}<0$, however there is no direct control over the surface dynamics. If $x_{i+1}$ is considered as the forcing term for the error surface dynamics, then the sliding condition outside some boundary layer is satisfied if $x_{i+1}=\bar{x}_{i+1}$ where

$$
\bar{x}_{i+1}=\dot{x}_{i d}-\hat{a}_{i} f_{i}-K_{i} S_{i}
$$

and the update law for the parameter estimate is as follows:

$$
\dot{\hat{a}}_{i}=\rho_{i} S_{i} f_{i} .
$$

where $K_{i}$ is a controller gain and $\rho_{i}$ is a positive gain. 
The next step is to force $x_{i+1} \rightarrow \bar{x}_{i+1}$, so define $S_{i+1}:=x_{i+1}-x_{i+1) d}$ where $x_{(i+1) d}$ equals $\bar{x}_{i+1}$ passed through a first-order filter,

$$
\tau_{i+1} \dot{x}_{(i+1) d}+x_{(i+1) d}=\bar{x}_{i+1}, \quad x_{(i+1) d}(0):=\bar{x}_{i+1}(0)
$$

After continuing this procedure for $1 \leq i \leq n-1$, define $S_{n}:=x_{n}-x_{n d}$. After taking its derivative, the control input is

$$
u=\dot{x}_{n d}-\hat{a}_{n} f_{n}\left(x_{1}, \ldots, x_{n}\right)-K_{n} S_{n}
$$

where $\dot{x}_{n d}$ is calculated from (10) and the update law of $\dot{\hat{a}}_{n}$ is following

$$
\dot{x}_{n d}=\frac{\bar{x}_{n}-x_{n d}}{\tau_{n}}, \dot{\hat{a}}_{n}=\rho_{n} S_{n} f_{n} .
$$

\subsection{Augmented Error Dynamics}

The augmented closed loop error dynamics is derived for analysis of stability and parameter convergence. After subtracting and adding $\bar{x}_{i+1}$ and $x_{(i+1) d}$, and using $u$ in (11), the closed-loop dynamics in (6) can be written as

$$
\begin{aligned}
& \dot{x}_{i}=\left[x_{i+1}-x_{(i+1) d}\right]+\left[x_{(i+1) d}-\bar{x}_{i+1}\right]+\bar{x}_{i+1}+a_{i} f_{i} \\
& \dot{x}_{n}=\dot{x}_{n d}-\hat{a}_{n} f_{n}-K_{n} S_{n}+a f_{n}
\end{aligned}
$$

for $i=1, \ldots, n-1$. Using (8) and the definition of error surfaces, the above equations can be described as a function of errors as follows:

$$
\begin{aligned}
& \dot{S}_{i}=S_{i+1}+\xi_{i+1}-K_{i} S_{i}+h_{i} \\
& \dot{S}_{n}=-K_{n} S_{n}+h_{n}
\end{aligned}
$$

where $\xi_{i+1}=x_{(i+1) d}-\bar{x}_{i+1}$ is the filter error and

$$
h_{i}=\left(a_{i}-\hat{a}_{i}\right) f_{i}=\tilde{a}_{i} f_{i}
$$

is the parameter estimation error multiplied by $f_{i}$.

In addition, we need to consider the augmented error dynamics due to inclusion of a set of the first order low pass filters and the update law for the estimate. After taking a derivative of $\xi_{i+1}$ for $1 \leq i \leq n-1$, the filter error dynamics is

$$
\dot{\xi}_{i+1}=\dot{x}_{(i+1) d}-\dot{\bar{x}}_{i+1}=-\xi_{i+1} / \tau_{i+1}-\dot{\bar{x}}_{i+1}
$$

where the last equality comes from (10). By taking a derivative of (8), we can write $\dot{\bar{x}}_{i+1}$ as

$$
\begin{gathered}
\dot{\bar{x}}_{2}=-\frac{d}{d t}\left(\hat{a}_{1} f_{1}\right)-K_{1} \dot{S}_{1} \\
\dot{\bar{x}}_{i+1}=\ddot{x}_{i d}-\frac{d}{d t}\left(\hat{a}_{i} f_{i}\right)-K_{i} \dot{S}_{i}=-\dot{\xi}_{i} / \tau_{i}-\frac{d}{d t}\left(\hat{a}_{i} f_{i}\right)-K_{i} \dot{S}_{i}
\end{gathered}
$$

for $i=2, \ldots, n-1$. Since the derivative of $h_{i}$ is written as

$$
\dot{h}_{i}=-\frac{d}{d t}\left\{\left(a_{i}-\hat{a}_{i}\right) f_{i}\right\}=-\frac{d}{d t}\left(\hat{a}_{i} f_{i}\right)+a_{i} \dot{f}_{i},
$$

(14) is rewritten as

$$
\begin{aligned}
& \dot{\bar{x}}_{2}=\dot{h}_{1}-a_{1} \dot{f}_{1}-K_{1} \dot{S}_{1} \\
& \dot{\bar{x}}_{i+1}=-\frac{\dot{\xi}_{i}}{\tau_{i}}+\dot{h}_{i}-a_{i} \dot{f}_{i}-K_{i} \dot{S}_{i}
\end{aligned}
$$

the filter error dynamics in (13) is

$$
\begin{gathered}
\dot{\xi}_{2}-K_{1} \dot{S}_{1}+\dot{h}_{i}=-\frac{\xi_{2}}{\tau_{2}}+a_{1} \dot{f}_{1} \\
\dot{\xi}_{i+1}-\frac{\dot{\xi}_{i}}{\tau_{i}}-K_{i} S_{i}+\dot{h}_{i}=-\frac{\xi_{i+1}}{\tau_{i+1}}+a_{i} \dot{f}_{i} \text { for } i=2, \ldots, n-1
\end{gathered}
$$

Equation (15) with the update law in (9) is written as

$$
\dot{h}_{i}=-\dot{\hat{a}}_{i} f_{i}+\tilde{a}_{i} \dot{f}_{i}=-\rho_{i} f_{i}^{2} S_{i}+\tilde{a}_{i} \dot{f}_{i}
$$

Finally, the overall error dynamics in (12), (17), and (18) can be summarized as

$$
\left\{\begin{aligned}
\dot{S}_{i} & =-K_{i} S_{i}+S_{i+1}+\xi_{i+1}+h_{i} \\
\dot{S}_{n} & =-K_{n} S_{n}+h_{n} \\
\dot{h}_{j} & =-\rho_{j} f_{j}^{2} S_{j}+\tilde{a}_{j} \dot{f}_{j} \\
\dot{\xi}_{2}-K_{1} \dot{S}_{1}+\dot{h}_{1} & =-\frac{\xi_{2}}{\tau_{2}}+a_{1} \dot{f}_{1} \\
\dot{\xi}_{k+1}-\frac{\dot{\xi}_{k}}{\tau_{k}}-K_{k} \dot{S}_{k}+\dot{h}_{k} & =-\frac{\xi_{k+1}}{\tau_{k+1}}+a_{k} \dot{f}_{k}
\end{aligned}\right.
$$

where $i=1, \ldots, n-1, j=1, \ldots, n$, and $k=2, \ldots, n-1$. Furthermore, the above error dynamics can be written in matrix form as follows:

$$
\begin{aligned}
{\left[\begin{array}{ccc}
I_{n} & 0 & 0 \\
0 & I_{n} & 0 \\
-K_{0} & I_{(n-1) \times n} & T_{\xi}
\end{array}\right]\left[\begin{array}{l}
\dot{S} \\
\dot{h} \\
\dot{\xi}
\end{array}\right]=} & {\left[\begin{array}{ccc}
A_{11} & I_{n} & I_{n \times(n-1)} \\
A_{21} & 0 & 0 \\
0 & 0 & -\Gamma
\end{array}\right]\left[\begin{array}{l}
S \\
h \\
\xi
\end{array}\right] } \\
& +\left[\begin{array}{cc}
0 & 0 \\
I_{n} & 0 \\
0 & I_{n-1}
\end{array}\right]\left[\begin{array}{c}
\tilde{a} \dot{f} \\
a \dot{f}
\end{array}\right]
\end{aligned}
$$

where the vectors are defined as

$$
\begin{gathered}
S=\left[S_{1} \cdots S_{n}\right]^{T} \in \mathfrak{R}^{n}, \xi=\left[\xi_{2} \cdots \xi_{n}\right]^{T} \in \mathfrak{R}^{n-1}, \\
h=\left[h_{1} \cdots h_{n}\right]^{T} \in \mathfrak{R}^{n}, \tilde{a} \dot{f}=\left[\tilde{a}_{1} \dot{f}_{1} \cdots \tilde{a}_{n} \dot{f}_{n}\right]^{T} \in \mathfrak{R}^{n}, \\
a \dot{f}=\left[a_{1} \dot{f}_{1} \cdots a_{n-1} \dot{f}_{n-1}\right]^{T} \in \mathfrak{R}^{n-1},
\end{gathered}
$$

and the submatrices are

$$
\begin{aligned}
& \left.A_{11}=\begin{array}{cccc}
-K_{1} & 1 & \cdots & 0 \\
0 & -K_{2} & \ddots & \vdots \\
\vdots & \vdots & \ddots & 1 \\
0 & 0 & \cdots & -K_{n}
\end{array}\right]=-K+\operatorname{diag}([1, \ldots, 1], 1) \in \mathfrak{R}^{n \times n}, \\
& A_{21}=-\operatorname{diag}\left(\rho_{1} f_{1}^{2}, \ldots, \rho_{n} f_{n}^{2}\right) \\
& K=\operatorname{diag}\left(K_{1}, \ldots, K_{n}\right), \quad K_{0}=\left[\operatorname{diag}\left(K_{1}, \ldots, K_{n-1}\right) 0_{n-1}\right] \in \mathfrak{R}^{(n-1) \times n}, \\
& \Gamma=\operatorname{diag}\left(\frac{1}{\tau_{2}}, \ldots, \frac{1}{\tau_{n}}\right) \in \mathfrak{R}^{(n-1) \times(n-1)}, \\
& T_{\xi}=I_{n-1}+\operatorname{diag}\left(\left[-\frac{1}{\tau_{2}}, \ldots,-\frac{1}{\tau_{n-1}}\right],-1\right) .
\end{aligned}
$$

Since the first block matrix in (20) is invertible such that

$$
\left[\begin{array}{ccc}
I_{n} & 0 & 0 \\
0 & I_{n} & 0 \\
-K_{0} & I_{(n-1) \times n} & T_{\xi}
\end{array}\right]^{-1}=\left[\begin{array}{ccc}
I_{n} & 0 & 0 \\
0 & I_{n} & 0 \\
T_{\xi}^{-1} K_{0} & -T_{\xi}^{-1} & T_{\xi}^{-1}
\end{array}\right]
$$

where

$$
T_{\xi}^{-1}=\left[\begin{array}{ccccc}
1 & 0 & \cdots & 0 & 0 \\
\frac{1}{\tau_{2}} & 1 & \cdots & 0 & 0 \\
\frac{1}{\tau_{2} \tau_{3}} & -\frac{1}{\tau_{3}} & \cdots & 0 & 0 \\
\vdots & \vdots & \ddots & \vdots & \vdots \\
\frac{1}{\tau_{2} \cdots \tau_{n-1}} & \frac{1}{\tau_{3} \cdots \tau_{n-1}} & \cdots & \frac{1}{\tau_{n-1}} & 1
\end{array}\right],
$$


after multiplying the inverse matrix to both sides in (20), the augmented closed-loop error dynamics are written as

$$
\dot{z}=A_{c l} z+\bar{B}_{w} \bar{\omega}
$$

where the error state $z=\left[S^{T} h^{T} \xi^{T}\right]^{T} \in \mathfrak{R}^{3 n-1}:=\Re^{n_{z}}$, $\bar{\omega}=\left[\begin{array}{ll}\tilde{a} \dot{f}^{T} & a \dot{f}^{T}\end{array}\right]^{T} \in \mathfrak{R}^{2 n-1}:=\mathfrak{R}^{n \omega}$, and the matrices are

$$
\begin{aligned}
A_{c l} & =\left[\begin{array}{ccc}
I_{n} & 0 & 0 \\
0 & I_{n} & 0 \\
T_{\xi}^{-1} K_{0} & -T_{\xi}^{-1} & T_{\xi}^{-1}
\end{array}\right]\left[\begin{array}{ccc}
A_{11} & I_{n} & I_{n \times(n-1)} \\
A_{21} & 0 & 0 \\
0 & 0 & -\Gamma
\end{array}\right] \\
& =\left[\begin{array}{ccc}
A_{11} & I_{n} & I_{n \times(n-1)} \\
A_{21} & 0 & 0 \\
A_{31} & A_{32} & A_{33}
\end{array}\right], \\
\bar{B}_{\omega} & =\left[\begin{array}{ccc}
0 & 0 \\
I_{n} & 0 \\
-T_{\xi}^{-1} I_{(n-1) \times n} & T_{\xi}^{-1}
\end{array}\right]
\end{aligned}
$$

where the submatrices are

$$
\begin{gathered}
A_{31}=T_{\xi}^{-1}\left(K_{0} A_{11}-I_{n}\right), \quad A_{32}=T_{\xi}^{-1} K_{0}, \\
A_{33}=T_{\xi}^{-1}\left(K_{0} I_{n \times(n-1)}-\Gamma\right)=T_{\xi}^{-1}\left(K_{1:(n-1)}-\Gamma\right), \\
K_{1:(n-1)}=\operatorname{diag}\left(K_{1}, \ldots, K_{n-1}\right) .
\end{gathered}
$$

It is noted that the third row of $\bar{B}_{w} \bar{\omega}$ is

$$
T_{\xi}^{-1}\left(-I_{(n-1) \times n} \tilde{a}+a\right) \dot{f}=T_{\xi}^{-1} \hat{a} \dot{f} .
$$

By defining $\omega=\left[\begin{array}{ll}\tilde{a} \dot{f}^{T} & \tilde{a} \dot{f}^{T}\end{array}\right]^{T},(21)$ is rewritten as follows:

$$
\dot{z}=A_{c l} z+B_{\omega} \omega
$$

where

$$
B_{w}=\left[\begin{array}{ll}
0 & 0 \\
I_{n} & 0 \\
0 & T_{\xi}^{-1}
\end{array}\right]
$$

Next, we need to determine the upper bound of $\omega$ in (22). Using the assumption in (7), the upper bound of $\omega_{i}$ for

$$
\begin{aligned}
& \left.\left|\omega_{i}\right|=\left|\tilde{a}_{i} \dot{f}_{i}\right|=\left|\tilde{a}_{i}\right|\left|\sum_{k=1}^{i} \frac{\partial f_{i}}{\partial x_{k}} \dot{x}_{k}\right|=\left|\tilde{a}_{i}\right||| \frac{\partial f_{i}}{\partial x_{1}} \cdots \frac{\partial f_{i}}{\partial x_{i}}\right]\left[\dot{x}_{1} \cdots \dot{x}_{i}\right]^{T} \mid \\
& \leq \lambda_{i} \gamma_{i}\left\|\left[\dot{x}_{1} \cdots \dot{x}_{i}\right]^{T}\right\| \\
& \left|\omega_{n+j}\right|=\left|\hat{a}_{j} \dot{f}_{j}\right|=\left|\hat{a}_{j}\right|\left|\sum_{k=1}^{j} \frac{\partial f_{j}}{\partial x_{k}} \dot{x}_{k}\right| \leq \lambda_{i} \gamma_{i}\left\|\left[\dot{x}_{1} \cdots \dot{x}_{i}\right]^{T}\right\| \\
& \leq \lambda_{i} \gamma_{i}\left\|\left[\dot{x}_{1} \cdots \dot{x}_{i}\right]^{T}\right\|
\end{aligned}
$$

for $j=1, \ldots, n-1$. Using (12), $\dot{x}_{i}$ is written in a function of $z$ as follows:

$$
\begin{aligned}
& \dot{x}_{1}=-K_{1} S_{1}+S_{2}+\xi_{2}+h_{1} \\
& \dot{x}_{i}=-K_{i} S_{i}+S_{i+1}+\xi_{i+1}+h_{i+1}-\xi_{i} / \tau_{i} \text { for } i=2, \ldots, n-1 \\
& \dot{x}_{n}=-K_{n} S_{n}-\xi_{n} / \tau_{n}
\end{aligned}
$$

Therefore, there exists a matrix $C_{z i}$ such that

$$
\left|\omega_{i}\right| \leq\left\|C_{z i} z\right\| .
$$

Finally, the augmented error dynamics, (19) with the upper bound of $\omega$ in (21), can be written in diagonal norm-bounded linear differential inclusion (LDI) form as follows[13]:

$$
\begin{aligned}
& \dot{z}=A_{c l} z+B_{\omega} \omega \\
& t_{i}=C_{z i} z,\left|\omega_{i}\right| \leq\left\|t_{i}\right\|, \quad i=1, \ldots, n_{\omega}
\end{aligned}
$$

\subsection{Quadratic Stability}

Since $A_{c l}$ in (24) is not time invariant due to $A_{21}$ is (21), both $A_{21}$ and $A_{31}$ can be decomposed into a steady-state term and a time varying term under the assumption that $z \rightarrow 0$ as $t \rightarrow \infty$ for the given set of controller gains. That is,

$$
\begin{aligned}
A_{21} & =-\operatorname{diag}\left(\rho_{1}, \ldots, \rho_{n}\right) \cdot \operatorname{diag}\left(f_{1}^{2}, \ldots, f_{n}^{2}\right) \\
& =-\Phi \cdot \operatorname{diag}\left\{f_{1 d}^{2}+\left(f_{1}^{2}-f_{1 d}^{2}\right), \ldots, f_{n d}^{2}+\left(f_{n}^{2}-f_{n d}^{2}\right)\right\} \\
& =A_{21}^{n}+A_{21}^{p} \\
A_{31} & =T_{\xi}^{-1}\left(K_{0} A_{11}-I_{(n-1) \times n} A_{21}\right) \\
& =T_{\xi}^{-1}\left(K_{0} A_{11}-I_{(n-1) \times n} A_{21}^{n}\right)-T_{\xi}^{-1} I_{(n-1) \times n} A_{21}^{p} \\
& =A_{31}^{n}+A_{31}^{p}
\end{aligned}
$$

where

$$
\begin{aligned}
& \Phi=\operatorname{diag}\left(\rho_{1}, \ldots, \rho_{n}\right), \\
& A_{21}^{n}=-\Phi \cdot \operatorname{diag}\left(f_{1 d}^{2}, \ldots, f_{n d}^{2}\right), \\
& A_{21}^{p}=-\Phi \cdot \operatorname{diag}\left(f_{1}^{2}-f_{1 d}^{2}, \ldots, f_{n}^{2}-f_{n d}^{2}\right), \\
& A_{31}^{n}=T_{\xi}^{-1}\left(K_{0} A_{11}-I_{(n-1) \times n} A_{21}\right), \\
& A_{31}^{p}=-T_{\xi}^{-1} I_{(n-1) \times n} A_{21}^{p}, \\
& f_{1 d}=f_{1}\left(x_{1 d}\right), \\
& f_{2 d}=f_{2}\left(x_{1 d},-\bar{a}_{1} f_{1 d}\right), \\
& f_{i d}=f_{i}\left(x_{1 d}, \ldots,-\bar{a}_{i} f_{i d}\right),
\end{aligned}
$$

and $\bar{a}_{i} \in\left[\begin{array}{ll}0 & c_{i}\end{array}\right]$ is a nominal constant, e.g., $\bar{a}_{i}=c_{i} / 2$ or a rough estimate of $a_{i}$. Therefore, $A_{c l}$ can be written as

$$
\begin{aligned}
A_{c l} & =\left[\begin{array}{ccc}
A_{11} & I_{n} & I_{n \times(n-1)} \\
A_{21}^{n} & 0 & 0 \\
A_{31}^{n} & A_{32} & A_{33}
\end{array}\right]+\left[\begin{array}{c}
0 \\
A_{21}^{p} \\
A_{31}^{p}
\end{array}\right]\left[\begin{array}{lll}
I & 0 & 0
\end{array}\right] \\
& =A_{n}+A_{p}
\end{aligned}
$$

Using (25), (24) can be considered as a nominal closed-loop error dynamics subject to a vanishing perturbation term as follows:

$$
\begin{aligned}
\dot{z} & =A_{c l} z+B_{w} \omega=\left(A_{n}+A_{p}\right) z+B_{w} \omega=A_{n} z+B_{w} \omega+B_{p} p \\
& =A_{n} z+\left[\begin{array}{ll}
B_{w} & B_{p}
\end{array}\right]\left[\begin{array}{l}
\omega \\
p
\end{array}\right]=A_{n} z+B_{e} \omega_{e}
\end{aligned}
$$

where $\omega_{e}=\left[\begin{array}{ll}\omega^{T} & p^{T}\end{array}\right]^{T} \in \mathfrak{R}^{n_{\omega}+n_{p}}:=\mathfrak{R}^{n_{e}}$,

$$
\begin{gathered}
B_{p} p=A_{p} z=\left[\begin{array}{c}
0 \\
A_{21}^{p} \\
A_{31}^{p}
\end{array}\right]\left[\begin{array}{lll}
I & 0 & 0
\end{array}\right] z= \\
{\left[\begin{array}{c}
0 \\
-\Phi \\
T_{\xi}^{-1} \Phi
\end{array}\right] \operatorname{diag}\left(f_{1}^{2}-f_{1 d}^{2}, \ldots, f_{n}^{2}-f_{n d}^{2}\right) S,} \\
p=\operatorname{diag}\left(f_{1}^{2}-f_{1 d}^{2}, \ldots, f_{n}^{2}-f_{n d}^{2}\right) S .
\end{gathered}
$$


Since $p$ is a function of $S$ and is bounded on $D$, there exists a matrix $C_{z i}$ such that $\left|p_{i}\right| \leq\left|C_{i z} z\right|$ for $i=2, \ldots, n-1$.

Finally, the augmented error dynamics in (24) can be written as

$$
\begin{aligned}
& \dot{z}=A_{n} z+B_{e} \omega_{e} \\
& t_{i}=C_{z i} z, \quad\left|\omega_{e i}\right| \leq\left\|t_{i}\right\|, \quad i=1, \ldots, n_{e}
\end{aligned}
$$

Since the augmented error dynamics in (27) is written in diagonal normbounded LDI, its quadratic stability can be applied as follows[13]:

Definition 1. Suppose $A_{n}$ in (27) is Hurwitz for the given set of controller gains, $\left\{K_{1}, \ldots, K_{n}, \tau_{2}, \ldots, \tau_{n}\right\}$, and update law gains, $\left\{\rho_{1}, \ldots, \rho_{n}\right\}$ The augmented error dynamics in (27) is quadratically stable if there exists a positive definite matrix $P$ such that

$$
\begin{aligned}
\frac{d}{d t} V(z) & =\frac{d}{d t}\left(z^{T} P z\right)= \\
\left(A_{n} z\right. & \left.+B_{e} \omega_{e}\right)^{T} P z+z^{T} P\left(A_{n} z+B_{e} \omega_{e}\right)<0 .
\end{aligned}
$$

If the error dynamics is quadratically stable, $z=0$ is an exponentially stable equilibrium point on $D$. The sufficient condition above for quadratic stability can be expressed in terms of linear matrix inequality (LMI) as described in the following theorem.

Theorem 1. Suppose that the diagonal norm-bounded error dynamics in (27) is given for given set of controller gains and $A_{n}$ is Hurwitz. The error dynamics in (27) is quadratically stable on $D$ if there exist $P>0$ and $\Sigma=\operatorname{diag}\left(\sigma_{1}, \sigma_{2}, \ldots, \sigma_{n_{e}}\right) \geq 0$ such that

$$
\left[\begin{array}{cc}
A_{n}^{T}+P A_{n}+C_{z}^{T} \Sigma_{B} C_{z} & P B_{e} \\
B_{e}^{T} P & -\Sigma
\end{array}\right]<0
$$

where

$$
C_{z}=\left[C_{z 1}^{T}, \ldots, C_{z n_{e}}^{T}\right]^{T}, \quad \Sigma_{B}=\operatorname{diag}\left(\sigma_{1} I, \sigma_{2} I, \ldots, \sigma_{n_{e}} I\right)
$$

is the diagonal block matrix.

The equivalence between (28) and (29) can be referred to[13].

Remark 1. If there exists the solution for (29), $z=0$ is exponentially stable. That is, $x_{1} \rightarrow x_{1 d}$ and $\tilde{a}_{i} f_{i} \rightarrow 0$ as $t \rightarrow \infty$. Moreover, if $f_{i}$ satisfies the so called "persistent excitation"'[6], i.e., there exist strictly positive constants $a_{i}$ and $T$ such that for any $t>0$,

$$
\int_{t}^{t+T} f_{i}^{2} d r \geq a_{i}
$$

$\tilde{a}_{i} \rightarrow 0$ as $t \rightarrow \infty$. Otherwise, it is not guaranteed for the estimated parameter to converge to the correct value although $x_{1} \rightarrow x_{1 d}$ as $t \rightarrow \infty$.

\section{Illustrative Example}

Consider (1) again with the unknown parameter $a=1$ and the control objective is $x_{1} \rightarrow x_{1 d}=1$. Suppose the domain $D=\left\{x \in \mathfrak{R}^{2}\|x\| \leq r, \quad r=2\right\}$ and $\hat{a} \in\left[\begin{array}{ll}0 & c\end{array}\right]$ where $c=2$. Then,

$$
\left|\frac{\partial f_{1}}{\partial x_{1}}\right|=\left|-2 x_{1}\right| \leq 2 r=\gamma,|\hat{a}| \leq c,|\tilde{a}| \leq c .
$$

If the controller derived in Section 1 is applied, the closed-loop error dynamics is following as in (19):

$$
\left\{\begin{aligned}
\dot{S}_{1} & =-K S_{1}+S_{2}+\xi+h \\
\dot{S}_{2} & =-K S_{2} \\
\dot{h} & =-\rho f_{1}^{2} S_{1}+\tilde{a} \dot{f}_{1} \\
\dot{\xi}-K \dot{S}_{1}+\dot{h} & =-\frac{\xi}{\tau}+a \dot{f}_{1} .
\end{aligned}\right.
$$

where $\xi=x_{2 d}-\bar{x}_{2}$ and $h=\tilde{a} f_{1}$. Equation (30) can be written in matrix form as follows:

$$
\left[\begin{array}{cccc}
1 & 0 & 0 & 0 \\
0 & 1 & 0 & 0 \\
0 & 0 & 1 & 0 \\
-K & 0 & 1 & 1
\end{array}\right] \dot{z}=\left[\begin{array}{cccc}
-K & 1 & 1 & 1 \\
0 & -K & 0 & 0 \\
-\rho f_{1}^{2} & 0 & 0 & 0 \\
0 & 0 & 0 & -1 / \tau
\end{array}\right] z+\left[\begin{array}{ll}
0 & 0 \\
0 & 0 \\
1 & 0 \\
0 & 1
\end{array}\right]\left[\begin{array}{c}
\tilde{a} \dot{f}_{1} \\
\dot{a} \dot{f}_{1}
\end{array}\right]
$$

where $z=\left[\begin{array}{llll}S_{1} & S_{2} & h & \xi\end{array}\right]^{T} \in \mathfrak{R}^{4}$. As derived in (22), (31) can be writtens as

$$
\begin{aligned}
\dot{z} & =\left[\begin{array}{cccc}
-K & 1 & 1 & 1 \\
0 & -K & 0 & 0 \\
-\rho f_{1}^{2} & 0 & 0 & 0 \\
-K^{2}+\rho f_{1}^{2} & K & K & K-1 / \tau
\end{array}\right] z+\left[\begin{array}{cc}
0 & 0 \\
0 & 0 \\
1 & 0 \\
0 & 1
\end{array}\right]\left[\begin{array}{c}
\tilde{a} f_{1} \\
\hat{a} \dot{f}_{1}
\end{array}\right] \\
& =A_{c l} z+B_{\omega} \omega .
\end{aligned}
$$

where $\omega=\left[\begin{array}{ll}\tilde{a}_{1} & \hat{a} \dot{f}_{1}\end{array}\right]^{T} \in \mathfrak{R}^{2}$ and the upper bound of $\omega$ is determined as follows:

$$
\begin{aligned}
& \left|\omega_{1}\right|=\left|\tilde{a} \dot{f}_{1}\right|=|\tilde{a}| \cdot\left|-2 x_{1} \dot{x}_{1}\right|=|\tilde{a}| \cdot\left|-2 x_{1}\right| \cdot\left|-K S_{1}+S_{2}+h+\xi\right| \\
& \quad \leq c \gamma\left|c_{z 1} z\right|=\left|C_{z 1} z\right| \\
& \left|\omega_{2}\right|=\left|\hat{a} \dot{f}_{1}\right|=|\hat{a}| \cdot\left|-2 x_{1} \dot{x}_{1}\right| \leq\left|C_{z 2} z\right|
\end{aligned}
$$

where $c_{z 1}=\left[\begin{array}{llll}-K & 1 & 1 & 1\end{array}\right]$ and $C_{z 1}=C_{z 2}=c \gamma c_{z 1}$.

Therefore, the augmented error dynamics can be written in LDI form as

$$
\begin{aligned}
& \dot{z}=A_{c l} z+B_{\omega} \omega \\
& t_{i}=C_{z i} z,\left|\omega_{i}\right| \leq\left|t_{i}\right|, \quad i=1,2
\end{aligned}
$$

For stability analysis, (32) is considered as a perturbed system as follows:

$$
\begin{aligned}
& \dot{z}=A_{c l} z+B_{\omega} \omega+B_{p} p \\
& t_{i}=C_{z i} z,\left|\omega_{i}\right| \leq\left|t_{i}\right|, \quad i=1, \quad 2
\end{aligned}
$$

where the matrices are

$$
A_{n}=\left[\begin{array}{cccc}
-K & 1 & 1 & 1 \\
0 & -K & 0 & 0 \\
-\rho f_{1}^{2}\left(x_{1 d}\right) & 0 & 0 & 0 \\
-K^{2}+\rho f_{1}^{2}\left(x_{1 d}\right) & K & K & K-1 / \tau
\end{array}\right], B_{p}=\left[\begin{array}{c}
0 \\
0 \\
-\rho \\
\rho
\end{array}\right],
$$

$p=\left[f_{1}^{2}\left(x_{1}\right)-f_{1}^{2}\left(x_{1 d}\right)\right] S_{1}$ is a vanishing perturbation and its upper bound is

$$
|p| \leq\left|f_{1}\left(x_{1}\right)+f_{1}\left(x_{1 d}\right)\right| \cdot\left|f_{1}\left(x_{1}\right)-f_{1}\left(x_{1 d}\right)\right| \cdot\left|S_{1}\right| \leq 2 M \cdot \gamma\left|S_{1}\right| \cdot\left|c_{z 2} z\right| \leq\left|C_{z 3} z\right|
$$
where $M$ is a maximum of $f_{1}$ on $D$, i.e., $M=r^{2}$,

$c_{z 2}=\left[\begin{array}{llll}1 & 0 & 0 & 0\end{array}\right]$ and $C_{z 3}=4 \gamma r M c_{z 2}$.

Finally, the augmented error dynamics is written in diagonal norm-bouned LDI as 


$$
\begin{aligned}
& \dot{z}=A_{n} z+\left[\begin{array}{ll}
B_{w} & B_{p}
\end{array}\right]\left[\begin{array}{c}
\omega \\
p
\end{array}\right]=A_{n} z+B_{e} e \\
& t_{i}=C_{z i} z, \quad\left|e_{i}\right| \leq\left|t_{i}\right|, \quad i=1,2,3
\end{aligned}
$$

The eigenvalues of $A_{n}$ in (34) are caculated as following:

$$
\begin{gathered}
\operatorname{det}\left(\lambda I-A_{n}\right)=\operatorname{det}\left(\left[\begin{array}{cccc}
\lambda+K & -1 & -1 & -1 \\
0 & \lambda+K & 0 & 0 \\
\rho f_{1 d}^{2} & 0 & \lambda & 0 \\
K^{2}-\rho f_{1 d}^{2} & -K & -K & \lambda-K+1 / \tau
\end{array}\right]\right) \\
=\operatorname{det}(\lambda+K) \operatorname{det}\left(\left[\begin{array}{ccc}
\lambda+K & -1 & -1 \\
\rho f_{1 d}^{2} & \lambda & 0 \\
K^{2}-\rho f_{1 d}^{2} & -K & \lambda-K+1 / \tau
\end{array}\right]\right)=0
\end{gathered}
$$

where $f_{1 d}=f_{1}\left(x_{1 d}\right)$. Then,

$$
\lambda+K=0, \tau \lambda^{3}+\lambda^{2}+K \lambda+\rho f_{1 d}^{2}=0
$$

It is noted that the decond characteristic equation can be derived using Symbolic Math Toolbox of MATLAB. Using the Routh stability criterion, the inequality condition for $A_{n}$ to be Hurwitz is derived to be

$$
K>0, K>\tau \rho f_{1 d}^{2}
$$

Suppose $K=2.5$ and $\tau=0.05$. Then, the inequality condition in (35) becomes

$$
\rho f_{1 d}^{2}<K / \tau=50
$$

If three values of $\rho$ are considered, i.e., $\rho_{i}=\{1,10,70\}, i=$ $1,2,3$, three ranges of $x_{1 d}$ for $A_{n}$ to be Hurwitz are obtained as follows:

$$
\left|f_{1}\left(x_{1 d}\right)\right|=\left|x_{1 d}^{2}\right|<\sqrt{50 / \rho_{i}} \Rightarrow\left|x_{1 d}\right|<\left(50 / \rho_{i}\right)^{1 / 4} .
$$

That is, $\left|x_{1 d}\right|<2.6591$ for $\rho_{1}=1,\left|x_{1 d}\right|<1.4953$ for $\rho_{2}=10$ and $\left|x_{1 d}\right|<0.9193$ for $\rho_{3}=70$.

When $x_{1 d}=1$ and $\rho_{i}$ is either 1 or 10 , the matrix $A_{n}$ in (32) is Hurwitz for both cases and LMI (29) can be solved via convex optimethod called CVX[14] is used to solve the feasibility problem by calculating $P$ and $\Sigma$ in (29) numerically in the framework of MATLAB. As predicted through stability analysis, $x_{1} \rightarrow x_{1 d}$ and $\hat{a} \rightarrow a$ as $t \rightarrow \infty$ as shown in Fig. 1. For $\rho_{3}=70$, the eigenvalues of $A_{n}$ are

$$
\lambda_{i}\left(A_{n}\right)=\{0.4134 \pm j 8.1884,-2.5,-20.8269\} \text {. }
$$

Since the matrix $A_{n}$ is not Hurwitz, this results in the oscillatory estimate of $a$ and thus oscillatory tracking of $x_{1 d}$ (refer to Fig 1).

If $x_{1 d}$ is changed to 1.5 , the eigenvalues of $A_{n}$ with respect to $\rho_{i}$ are

$$
\begin{aligned}
& \lambda\left(A_{n}\right)=\{-1.2652 \pm j 2.0482,-2.5,-17.4697\} \text { for } \rho=1, \\
& \lambda\left(A_{n}\right)=\{0.0139 \pm j 7.1102,-2.5,-20.0277\} \text { for } \rho=10 .
\end{aligned}
$$

It is shown in Fig. 2 that the time responses of for $x_{1}$ and $\hat{a}$ are oscillatory for $\rho_{2}$ while the tracking error and parameter estimation error converges to zero for $\rho_{1}$. If $\tau$ becomes smaller as 0.01 , the inequality condition in (36) is modified as

$$
\left|x_{1 d}\right|<\left(250 / \rho_{i}\right)^{1 / 4} \text {. }
$$

Thus, the matrix $A_{n}$ is Hurwitz for all $\rho_{i}$ and there exist a solution for LMI (29). The corresponding time responses of $x_{1}$ and $\hat{a}$ are shown in Fig. 3. It is remarked that a smaller gain of $\tau$ allows us to enlarge the range of $x_{1 d}$ for $A_{n}$ to be Hurwitz. However, it is well known that $1 / \tau$ in the first-order filter is a cut-off frequency and the noise thus may not be attenuated if $\tau$ is too small.
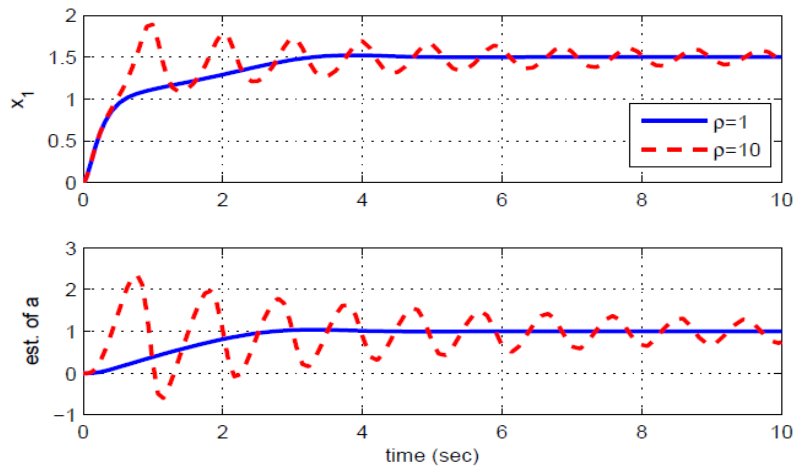

Figure 2. Time response of $x_{1}$ and $\hat{a}$ with respect to $\rho$ for $x_{1 d}=1.5$
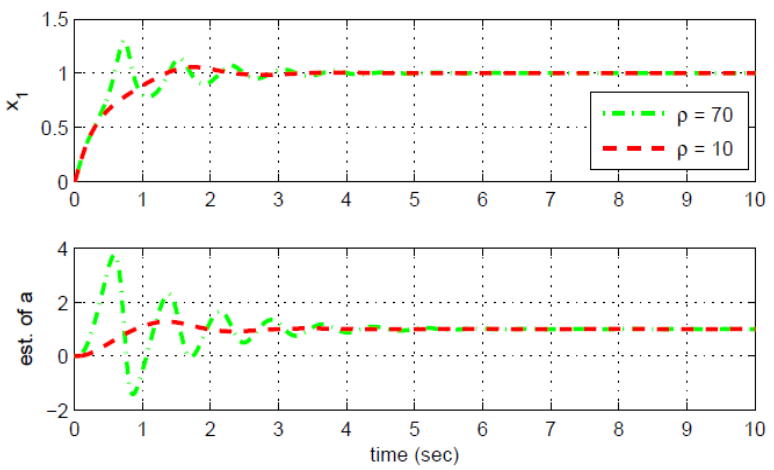

Figure 3. Time response of $x_{1}$ and $\hat{a}$ with respect to $\rho$ for $\tau=0.01$

\section{Conclusions}

The analysis method for stability and parameter convergence of adaptive dynamic surface control was proposed by deriving the augmented closed-loop error dynamics in linear differential inclusion form. The sufficient condition for stability is derived for the given controller gains in the form of linear matrix inequality. It allows us to analyze both quadratic stability and parameter convergence by computing a quadratic Lyapunov function numerically via convex optimization.

\section{ACKNOWLEDGEMENTS}

This research was supported by the Industrial Strategic Technology Development Program (10035250, Development of Spatial Awareness and Autonomous Driving Technology for Automatic Valet Parking) funded by the Ministry of Knowledge Economy (MKE, Korea).

\section{REFERENCES}


[1] D. Swaroop, J. K. Hedrick, P. P. Yip, J. C. Gerdes, "Dynamic surface control for a class of nonlinear systems", IEEE Transactions on Automatic Control, vol. 45, no. 1, pp. 1893-1899, 2000.

[2] B. Song, J. K. Hedrick, Dynamic surface control of uncertain nonlinear systems: an LMI approach, Springer, 2011.

[3] M. Krstic, I. Kanellakopoulos, P. Kokotovic, Nonlinear and adaptive control design, Wiley Interscience, 1995.

[4] S. K. Nguang, "Robust stabilization of a class of time-delay nonlinear systems", IEEE Transactions on Automatic Control, vol. 45, no. 4, pp. 756-762, 2000.

[5] S. S. Ge, F. Hong, T. H. Lee, "Robust adaptive control of nonlinear systems with unknown time delays", Automatica, vol. 41, pp. 1181-1190, 2005.

[6] T. P. Hedrick, P. P. Yip, “Adaptive dynamic surface control: a simplified algorithm for adaptive backstepping control of nonlinear systems", International Journal of Control, vol. 71, no. 5, pp. 959-979, 1998.

[7] S. J. Yoo, J. B. Park, Y. Ho. Choi, "Adaptive dynamic surface control for stabilization of parametric strict-feedback nonlinear systems with unknown time delays", IEEE Transactions on Automatic Control, vol. 52, no. 12, pp. 2360-2365, 2007.

[8] T. P. Zhang, S. S. Ge, “Adaptive dynamic surface control of nonlinear systems with unknown dead zone in pure feedback form”, Automatica, vol. 44, pp. 1895-1903, 2008.

[9] Q. Zhao, Y. Lin, "Adaptive dynamic surface control fure-feedback systems", Int. J. Robust and Nonlinear Control, doi: 10.1002/rnc.1774, 2011.

[10] J.-J. Slotine, W. Li, Applied Nonlinear systems, $3^{\text {rd }}$ Edition, Prentice Hall, 1991.

[11] H. K. Khalil, Nonlinear Systems, $3^{\text {rd }}$ Edition, Prentice Hall, 2002.

[12] J. K. Hedrick, P. P. Yip, "Multiple sliding surface control: theory and application", J. Dynamic Systems, Measurement, and Control, vol. 122, pp. 586-593, 2000.

[13] S. Boyd, 1. El Ghaoui, E. Feron, V. Balakrishan, Linear Matrix Inequalities in System and Control Theory, SIAM, 1994.

[14] M. Grant, S. Boyd, "CVX: Matlab software for disciplined convex programming", version 1.21, http://cvxr.com/cvx. 